\title{
Reducing the severity of stroke
}

\author{
Georgina Meredith, Anthony Rudd
}

Stroke Unit, St Thomas Hospital, London, UK

\section{Correspondence to}

Dr Anthony Rudd, Stroke Unit, St Thomas Hospital, London SE1 7EH, UK;

anthony.rudd@kcl.ac.uk

Received 2 February 2019

Revised 3 April 2019

Accepted 8 April 2019

Published Online First 25 May 2019

\section{ABSTRACT}

Stroke remains one of the most important causes of death and disability worldwide. Effective prevention could reduce the burden of stroke dramatically. The management of stroke has undergone a revolution over the last few decades, particularly with the development of techniques for revascularisation of patients with ischaemic stroke. Advanced imaging able to identify potentially salvageable brain is further increasing the potential for effective acute treatment. However, the majority of stroke patients won't benefit from these treatments and will need effective specialist stroke care and ongoing rehabilitation to overcome impairments and adapt to living with a disability. There are still many unanswered questions about the most effective way of delivering rehabilitation. Likewise, research into how to manage primary intracerebral haemorrhage has yet to transform care.

\section{INTRODUCTION}

Up until the 1990s stroke was widely believed to be a largely untreatable condition with no major advances in management having been found since William Osler wrote at the beginning of the 20th century that 'it is the duty of the physician to explain to the patient or to his friends, that the condition is past relief, that medicines and electricity will do no good and there is no possible hope of cure'. ${ }^{1}$

The last 30 years have seen a dramatic change in that view. Not only is it recognised that stroke is largely preventable but for many patients who suffer a stroke, there are treatments that can transform them from having a life of long-term disability to one of independence. Nevertheless, there are unfortunately still many patients left, after the acute phase of the illness, with impairments requiring skilled rehabilitation and there is a dearth of high-quality evidence to show how they should be managed. This article highlights the important advances that have been made and where more research is needed.

\section{The burden of stroke}

It is estimated that there are 4.5 million deaths from stroke a year worldwide and over 9 million survivors. ${ }^{2}$ The estimated global lifetime risk of stroke for the population aged over 25 is about $25 \%$ for both men and women, with the risk of ischaemic stroke being about $18 \%$ and about $8 \%$ for haemorrhage. However, there are big regional variations with the risk being about 39\% in East Asia and only $12 \%$ in east sub-Saharan Africa. ${ }^{3}$ Even though in high-income countries incidence and case fatality have fallen dramatically over the last 20 years, because of an ageing population it is estimated that over the next 20 years in Europe and therefore, likely to be mirrored in other high-income countries, there will be $45 \%$ more deaths caused by stroke, an extra 1 million stroke survivors and $32 \%$ more disability-adjusted life years lost. ${ }^{4}$ Costs to society resulting from stroke are huge. In the UK alone, it is estimated that stroke costs about $£ 7$ billion per year. ${ }^{5}$ Stroke is an expensive disease because over half of the survivors will have a significant disability resulting in long-term health and social care costs, loss of earnings and costs to the family. So, for everyone, the risk of stroke is high and the impact on society substantial highlighting the importance of effective prevention, good acute care and rehabilitation.

\section{Prevention of stroke}

Stroke is not a single disease but rather an all-encompassing term for focal vascular disease in the brain. It is, therefore, vital that the diagnostic process does not stop at stroke but rather identifies the type of stroke and therefore, its likely aetiology. The Trial of Org 10172 in Acute Stroke Treatment (TOAST) classification is the most widely used system for defining the likely underlying cause. ${ }^{67}$ TOAST classifies stroke as being due to:

- thrombosis or embolism due to atherosclerosis of a large artery $(15.3 \%$ of cases in European populations),

- embolism of cardiac origin $30.2 \%$ of cases in European populations),

- occlusion of a small blood vessel $(25.8 \%$ of cases in European populations),

- other determined cause $2.1 \%$ of cases in European populations) and

- undetermined cause (two possible causes, no cause identified or incomplete investigation) (39.3\% of cases in European populations).

The most effective means available for reducing the burden of stroke involve modification and treatment of vascular risk factors. ${ }^{8}$ Hypertension is the most prevalent vascular risk factor for stroke at about $30 \%$ in the UK and the USA in people aged over 16 years. ${ }^{9}$ The risk of death from stroke increases progressively and linearly with systolic blood pressure levels as low as $115 \mathrm{~mm} \mathrm{Hg}$ and diastolic 75 mm Hg upwards. ${ }^{10}$ For every $20 \mathrm{~mm} \mathrm{Hg}$ systolic or $10 \mathrm{~mm} \mathrm{Hg}$ diastolic increase in blood pressure, there is a doubling of mortality from stroke.

Atrial fibrillation (AF) increases the relative risk of stroke 2.6-4.5-fold. In patients with AF, treated with adjusted-dose warfarin or a direct oral anticoagulant (DOAC), the relative risk reduction of stroke is $68 \%$ (95\% CI, 50 to 79$)$ and the absolute annual risk is reduced from $4.5 \%$ to $1.4 \% .^{11}$

Diabetes increases the relative risk of stroke from 1.8 to $6.0 .^{12}$ The UK Prospective Diabetes Study has 
Box 1 Recommendations for reducing the risk of stroke

- Antiplatelet agents should be used for patients with cerebral infarction. Use aspirin $300 \mathrm{mg}$ daily for 2 weeks and then substitute for long-term clopidogrel $75 \mathrm{mg}$ thereafter. If there are concerns about affordability or clopidogrel resistance or intolerance, it is reasonable to continue with aspirin $75 \mathrm{mg}$ long-term.

- Blood pressure management. Probably the lower the blood pressure the better so most patients will benefit from antihypertensive treatment.

- For patients in atrial fibrillation, anticoagulation should be started 2 weeks after the cerebral infarct. If the stroke is small and the patient is being discharged from hospital then earlier commencement is appropriate. Aspirin or clopidogrel is not an alternative to anticoagulation. Warfarin and the direct oral anticoagulants (DOACs) are equally effective but most patients prefer the convenience of taking DOACs.

- Lipids. The decision to initiate treatment should be determined by a person's absolute cardiovascular risk rather than their cholesterol level. Use a 'high-intensity' statin, such as atorvastatin $80 \mathrm{mg}$ daily, with a lower starting dose for people at high risk of adverse events or drug interactions. ${ }^{57}$

- Smoking. Give advice and support for cessation.

- Provide advice on diet and exercise.

shown that rigorous glycaemic control through lifestyle change and pharmacological treatment can reduce the microvascular events of nephropathy, retinopathy and peripheral neuropathy associated with diabetes, but, the effects on reducing macrovascular diseases, such as stroke and myocardial infarction, are less clear.

Lowering blood lipid levels, especially low-density lipoprotein (LDL) using statins, has been shown to reduce the relative risk of stroke anywhere between $10 \%$ and $50 \%$ in several randomised controlled trials. ${ }^{13}$ In primary and secondary prevention studies, lowering LDL cholesterol by $1 \mathrm{mmol} / \mathrm{L}$ reduces the relative risk of major vascular events by $21 \%$, total mortality by $9 \%$ and stroke (of any type) by $15 \%$ irrespective of baseline cholesterol and gender. ${ }^{14}$

Smoking is an independent stroke risk factor, increasing the risk of stroke by about $50 \% .{ }^{15}$ The risk increases proportionally with the number of cigarettes smoked per day and passive smoking also increases the risk of ischaemic stroke.

Antiplatelet medication is not indicated for patients who have had no previous vascular events. For secondary prevention, after both TIA and stroke, the evidence supports the use of aspirin alone, clopidogrel alone or combined aspirin and dipyridamole-modified release. ${ }^{16}$ There have been no trials supporting the use of cilostazol or any of the newer antiplatelet drugs commonly used for ischaemic heart disease and there are few indications for combining aspirin and clopidogrel (box 1).

\section{Acute stroke interventions}

The recent evidence about the benefits of thrombectomy have dominated the agenda for stroke over the last few years. It is, however, important to put into context the impact of acute treatments so as not to forget that the most important intervention remains the careful delivery of high-quality basic multidisciplinary care (box 2).
Box 2 For every 100 patients treated

- Up to $15 \%$ suitable for intravenous thrombolysis. Number needed to treat (NNT) for one good outcome (modified Rankin score of less than 2)=7-two patients benefit.

- Up to $10 \%$ suitable for thrombectomy. NNT of between 2 and 7-two to five patients benefit.

- About $30 \%$ will spontaneously recover with good basic medical care-30 patients benefit.

- About $10 \%$ will die in the first week even with high-quality care-10 patients death inevitable.

- More than 50 patients will be left with a disability and need ongoing treatment on a stroke unit.

\section{SPECIALIST MULTIDISCIPLINARY STROKE CARE-STROKE UNITS AND EARLY SUPPORTED DISCHARGE}

In the past 25 years, the medical care of patients with stroke has changed enormously in high-income countries. The central change has been the development of organised systems of stroke care, characterised by a move away from general medicine towards specialised multidisciplinary models of care based on the stroke unit model. Randomised controlled trials have shown that being admitted to a stroke unit improves survival and reduces long-term dependency. In the most recently updated Cochrane review of organised stroke care summarising the results of 28 trials, the odds of death or dependency at 1 year were reduced by $19 \%$ and $21 \%$, respectively. ${ }^{17}$ Stroke units have been evaluated in many different countries and settings, and found to be effective in all types of stroke patient and in both high-income and middle/low-income countries. ${ }^{18}$ The trials have been conducted over 20th century years are very similar to those from the 21st century. The benefits are, therefore, not solely to do with the delivery of reperfusion treatments, which have only been available for the last 20 years. The key elements of stroke unit care are described in a paper by Langhorne and Pollock. ${ }^{19}$

The principles of specialist organised stroke care have been extended to provide early supported discharge services, where multidisciplinary care and therapy are provided in the patient's own home at a similar intensity to inpatient rehabilitation. Meta-analysis shows improved long-term recovery and shorter length of hospital stay. ${ }^{20}$

\section{ACUTE REPERFUSION}

For patients with ischaemic stroke, early treatment to restore blood flow to the affected area of the brain can limit the extent of damage and increase the patient's chance of making a recovery. Reperfusion can be achieved either through the administration of a thrombolytic drug or through intra-arterial clot retrieval. The first landmark trial to demonstrate the effectiveness of thrombolysis was the NINDS trial in $1995,{ }^{21}$ which found that the drug alteplase significantly reduced the rate of disability if given within 3 hours of stroke onset. Thrombolysis has since been evaluated in a number of trials and has been shown to improve recovery up to 4.5 hours after the onset of symptoms in selected patients ${ }^{22}$ (figure 1). $\chi$

Few countries have achieved rates of thrombolysis greater than $15 \%$ of stroke admissions with the main limiting factors being delayed arrival at the hospital and stroke occurring during sleep without a known time of onset. This proportion will only increase substantially if there is a combination of both greater public awareness of the symptoms of stroke and the urgency of seeking medical attention and better organisation of prehospital 
Reviฆw: Thrombolysis br acuts ischasmic stoles

Comparison: 1 Any thrombolyic agent vereus ontol

Qubome: 12 Alve and independent (mRS o to 2 ) at end of blow-up, paricipants teated up to ax hours

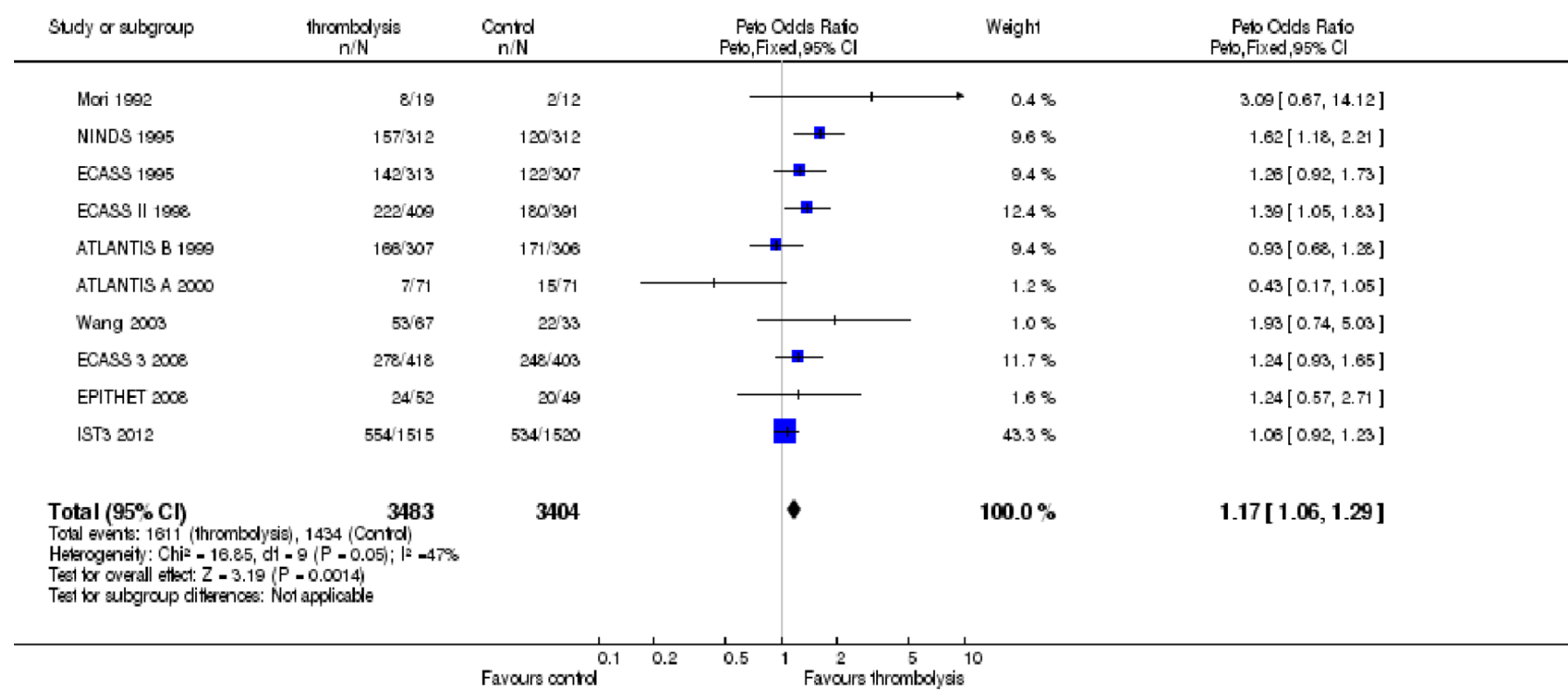

Figure 1 Summary of meta-analysis of trials of intravenous thrombolysis after stroke. Outcome being alive and independent (mRS 0-2) at the end of follow-up after treatment up to 6 hours after the onset of symptoms. mRS, modified Rankin score.

and emergency room care. Public awareness campaigns, such as the FAST campaign, run on an annual basis in the UK can be effective, although the effect seems to be short-lived. ${ }^{23}$

Barriers to immediate treatment need to be removed. Treatment should take precedence over sorting out payment. Where a patient cannot give verbal consent to treatment then the physician should be able to make the decision on their behalf.

The WAKE-UP trial published in 2018 did show that patients who had developed a stroke during sleep but with evidence that there was salvageable brain did benefit from treatment with alteplase. Patients had to have restricted diffusion on MRI but no corresponding abnormality on flair imaging, suggesting that the onset of symptoms was within the previous 4.5 hours. The trial excluded patients suitable for intra-arterial clot retrieval and does suggest that, maybe, the belief that stroke occurring during sleep does not cause the patient to wake may be erroneous. ${ }^{24}$

Intra-arterial clot retrieval is now clearly established as an option for a small proportion of patients. The defining trial, MR CLEAN, conducted in Holland and published in 2015 showed for the first time, after multiple negative studies, that thrombectomy using modern retrieval devices within 6 hours of the onset of symptoms was highly effective. ${ }^{25}$ This was quickly followed by a series of other trials all showing similar results summarised in the Hermes meta-analysis. ${ }^{26}$ The number needed to treat (NNT) is 2.6 to reduce the disability level by at least one point on the modified Rankin score at 90 days. As with the intravenous thrombolysis trials, there was no effect on the risk of death. Further studies have shown that using advanced imaging techniques identifying whether there is a significant ischaemic penumbra can extend the benefit of thrombectomy to patients up to 24 hours after the onset of symptoms. ${ }^{27} 28$

It is estimated that approximately $10 \%$ of patients with ischaemic stroke might benefit from thrombectomy. They must have evidence of occlusion of the internal carotid or M1 or M2 segments of the middle cerebral artery, present within 6 hours, unless imaging suggests salvageable brain beyond this time and have been previously independent. There is little randomised controlled trial evidence of benefit for patients with anterior cerebral artery occlusion or posterior circulation, although many clinicians treat these patients despite the lack of evidence. Countries vary in their capacity to deliver this treatment with the major limiting factor being the lack of skilled interventional neuroradiologists able to provide full geographical coverage 24 hours a day. In the UK, in 2018, only about $1 \%$ of stroke admissions received thrombectomy. ${ }^{29}$ Development of these services must be a major priority for all health services.

Large areas of infarction may cause significant cytotoxic oedema resulting in what is termed 'malignant middle cerebral artery syndrome'. Oedema secondary to infarction does not respond to steroids or trials of mannitol and other drugs aiming to reduce oedema are ineffective. ${ }^{30}$ Under these circumstances, surgical decompression using hemicraniectomy may be the only way of improving outcome. ${ }^{31} 32$

\section{Prevention and management of complications}

Most patients dying of acute stroke do not die directly from brain injury, but complications, such as aspiration pneumonia and pulmonary embolism. Being managed in a stroke unit, nursed by experts in the disease, able to prevent and if necessary rapidly recognise developing complications, probably explains much of their benefit. It has been shown using data from the England National Stroke Audit that nurse staffing levels are associated with risk of death. Having less than three nurses on duty at any time for every 10 beds significantly increases 30-day mortality after acute stroke ${ }^{33}$ (figure 2).

Likewise, the processes of care, such as time to consultant assessment, time to screening for dysphagia and particularly, provision of early hydration and aspirin to patients with ischaemic stroke, have a major impact on mortality, ${ }^{3435}$ (figures 3 and 4). A trial in Australia implementing evidence-based treatment protocols for fever, hyperglycaemia and swallowing dysfunction showed dramatic improvements in outcomes. ${ }^{36}$ 


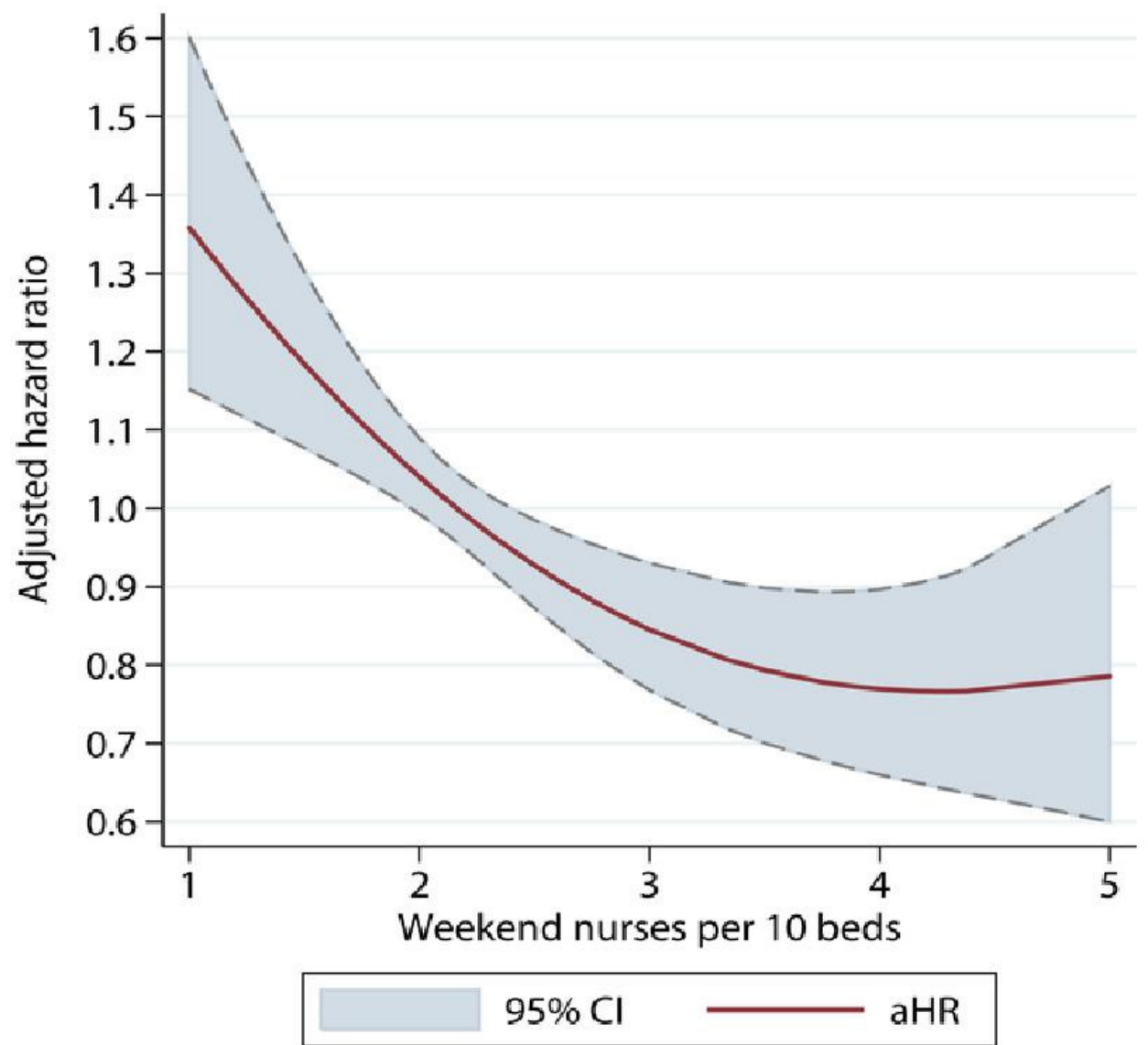

Figure 2 Nurse staffing levels: Adjusted Hazard Ratio of 30-day Mortality of Patients Admitted on Weekends, by Ratio of Registered Nurses Per Ten Beds on the Weekend

One of the major causes of morbidity and mortality after stroke is venous thromboembolism (VTE) ${ }^{37}$ One study using direct thrombus imaging after stroke has shown that about $50 \%$ of hemiplegic legs develop venous thrombosis, although not all above knee deep venous thrombosis (DVT). ${ }^{38}$ Trials using low-dose anticoagulation, while reducing the risk of DVT, increases the risk of haemorrhagic transformation of infarction ${ }^{39}$ and is, of course, contraindicated in primary intracerebral haemorrhage. The CLOTS 1 and 2 trials $^{4041}$ testing the use of shortlength and full-length antiembolism stockings showed they were ineffective at preventing VTE. However, CLOTS $3^{42}$ showed that using intermittent compression devices for the first 30 days after a stroke or until the patient was discharged or regained mobility did significantly reduce both VTE and death (figure 5).

\section{Acute management of intracerebral haemorrhage}

Primary intracerebral haemorrhage accounts for about $10 \%$ of stroke. Compared with ischaemic stroke relatively little progress has been made in improving outcomes. Rebleeding is the

\section{"Stroke unit" item}

Early stroke consultant assessment

CT scan within 24 hours

Early nurse \& therapist assessment

Early swallow assessment \& nutrition management

Early $i v$ fluids and aspirin

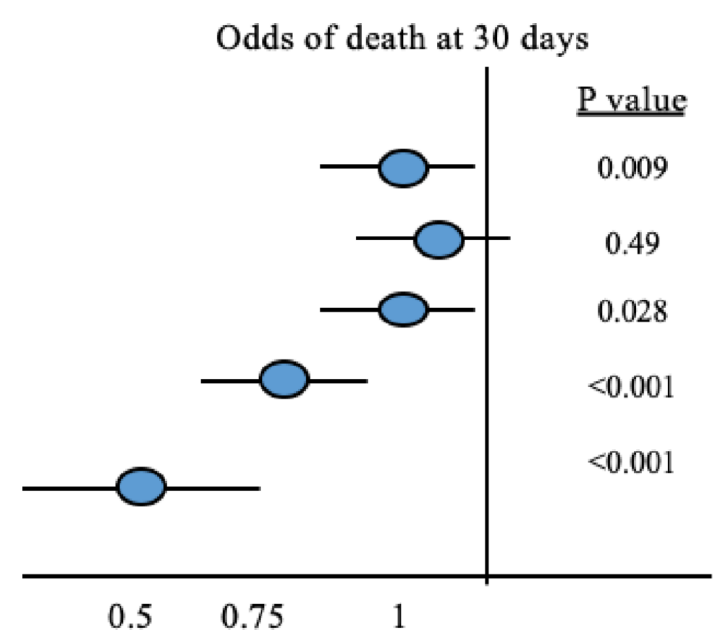

Figure 3 Impact of care processes on odds of death at 30 days after stroke 


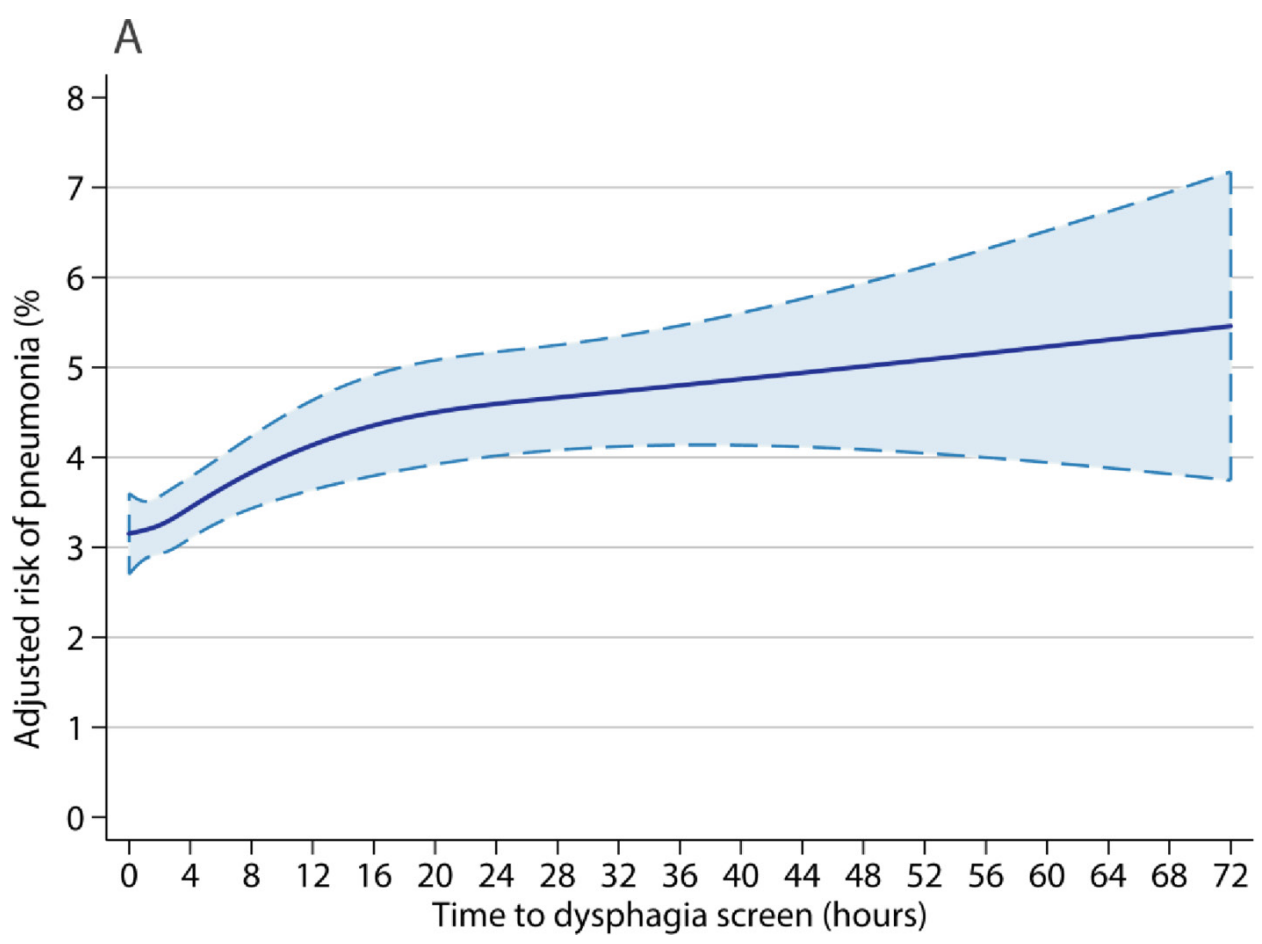

Figure 4 Time to dysphagia screen and risk of stroke-associated pneumonia

most common reason for deterioration and death. ${ }^{43}$ Recent research has shown that rapid, careful blood pressure control and reversal of anticoagulation are important. The INTERACT2 trial showed that reducing blood pressure to $140 \mathrm{~mm} \mathrm{Hg}$ systolic reduced disability with an NNT of 13 but had no impact on mortality. ${ }^{44}$ The ATACH-2 trial tested whether lowering blood pressure to $120 \mathrm{~mm} \mathrm{Hg}$ was beneficial but failed to show any benefit compared with the control group, which achieved a median systolic blood pressure of $140 \mathrm{~mm} \mathrm{Hg}$, which was the level achieved in the intervention group of INTERACT2. The message is that blood pressure should be reduced to $140 \mathrm{~mm}$ $\mathrm{Hg}$ but not lower, possibly because lowering further reduces cerebral blood flow to the penumbra surrounding the haemorrhagic area of brain. ${ }^{45}$

With better detection and management of patients with AF, there are increasing numbers of patients being admitted with intracerebral haemorrhage secondary to anticoagulation treatment. Rapid reversal of anticoagulants using prothrombin complex concentrate and vitamin $\mathrm{K}$ for patients on warfarin, idarucizumab for dabigatran and andexxa for apixaban and rivaroxaban is essential. ${ }^{46}$ Having a care bundle to address all these issues including when to refer to neurosurgery improves mortality. ${ }^{47}$ Surgery after haemorrhage is only indicated where a patient has a deteriorating level of consciousness, significantly

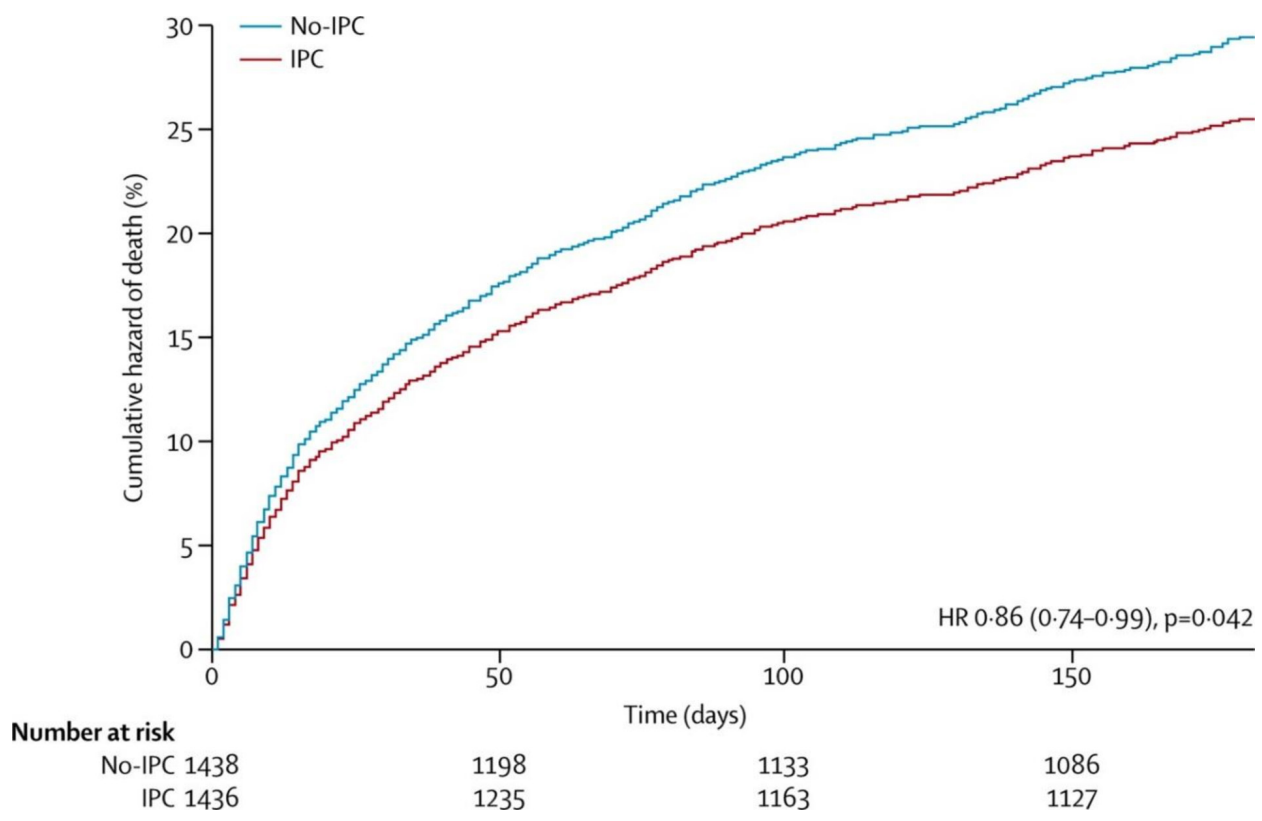

Figure 5 Risk of death after stroke treated with Intermittent Pneumatic Compression versus control 
Current research questions

1. How do we implement what we know about reducing the risk of stroke more effectively in the population?

2. How do we ensure that patients get to a centre able to deliver high quality of care as soon as possible after the onset of symptoms?

3. What can be done to prevent rebleeding after a primary intracerebral haemorrhage?

4. How does one maximise neurological recovery after stroke?

5. What is the ideal intensity of therapy and what techniques are most successful for different types of impairment?

\section{Key references}

1. Sacco R, Adams R, Albers $G$, et al. Guidelines for prevention of stroke in patients with ischemic stroke or transient ischemic attack: a statement for healthcare professionals from the American Heart Association/American Stroke Association Council on Stroke: co-sponsored by the Council on Cardiovascular Radiology and Intervention: the American Academy of Neurology affirms the value of this guideline. Stroke 2006;37(2):577-617.

2. National Clinical Guidelines for Stroke (Intercollegiate Stroke Working Party 5th edition 2015 Royal College Physicians, London). https://www.strokeaudit.org/SupportFiles/ Documents/Guidelines/2016-National-Clinical-Guideline-forStroke-5t-(1).aspx.

3. Stroke Unit Trialists' Collaboration, 2013. https://www.cochrane.org/CD000197/ STROKE_organised-inpatient-stroke-unit-care.

4. Cochrane review. Clot-dissolving drugs for treating ischaemic stroke in the early stages 2014. https://www.cochrane.org/ CD000213/STROKE_clot-dissolving-drugs-treating-ischaemicstroke-early-stages.

5. Goyal M, Menon BK, van Zwam WH, et al. Endovascular thrombectomy after large-vessel ischaemic stroke: a metaanalysis of individual patient data from five randomised trials. Lancet 2016;387(10029):1723-31. doi: 10.1016/S01406736(16)00163-X. Epub 18 February 2016.

raised intracranial pressure or hydrocephalus. Trials of routine surgery for haemorrhage compared with the best medical treatment have shown no benefit. ${ }^{48} 49$

\section{Organisation of acute stroke care}

There is some evidence to suggest that larger stroke services work more effective than small ones ${ }^{34}$ and certainly where the workforce is limited, it makes sense to centralise acute stroke care into a smaller number of larger units. This has been achieved in London, UK, which transformed services such that 32 acutely admitting units were reduced to just 10 delivering the first 72 hours of care for all patients in the city before transferring on those that needed ongoing bed-based stroke unit care to 1 of a further 16 units. At the same time, as London underwent redesign of stroke care, changes were also made in Manchester; however, only centralising patients possibly suitable for intravenous thrombolysis. Comparing the two models with each other and against the background of changes over time in England as a whole showed that there were significant reductions in mortality (estimated 96 lives saved per annum), length of stay and overall
Self-assessment questions

1. Risk factors for ischaemic stroke include
a. Hypertension
b. Atrial fibrillation
c. Low total cholesterol
d. Air pollution
e. Female gender

2. The following drugs have been shown to be effective at reducing the risk of recurrent stroke
a. Aspirin
b. Clopidogrel
c. Dipyridamole
d. Ezetimibe
e. Statins

3. Evidence supports the use of intra-arterial clot retrieval (thrombectomy) for

a. Occlusion of the M3 segment of the middle cerebral artery

b. Vertebral artery occlusion

c. Patients taking anticoagulants

d. Patients with significant prior disability (Rankin 3 and above) but who have presented with significant additional impairment secondary to a large vessel occlusion

e. Patients within 6 hours of onset of symptoms and occlusion of the proximal middle cerebral artery

4. For every 100 patients with ischaemic stroke

a. Approximately 10 will be suitable for thrombectomy

b. Approximately 30 will be suitable for intravenous thrombolysis

c. Approximately 50 will benefit from stroke unit care

d. Approximately 20 will have a prior history of hypertension

e. Approximately 20 will have atrial fibrillation as the cause

5. Patients with primary intracerebral haemorrhage

a. Will usually need surgery to remove the blood

b. Should have their blood pressure lowered to a maximum of $120 \mathrm{~mm} \mathrm{Hg}$ systolic as soon as possible after presentation

c. Will usually have an underlying structural abnormality, such as an arteriovenous malformation

d. Lobar haemorrhages in older patients are often due to amyloid angiopathy

e. Basal ganglia haemorrhages are most commonly associated with hypertension

costs by centralising care but only in the London model and not where the only change was targeted at thrombolysis. ${ }^{50-52}$

\section{Rehabilitation}

Helping people to recover, regain function and return to doing the activities and work they were doing before their stroke is essential to reduce the severity of stroke. The problems needing support and rehabilitation are not just the obvious ones of impaired mobility, speech and activities of daily living but depression, anxiety, visual and perceptual deficits, cognitive impairments, fatigue and many others ${ }^{53}$.

Compared with other areas of stroke care, however, there have been few large clinical trials and a relatively weak evidence base. These are summarised in the National Clinical Guidelines for Stroke, fifth edition. ${ }^{54}$ Drugs given to enhance recovery have so far proved disappointing with the study testing fluoxetine in the FOCUS trial ${ }^{55}$ showing no benefit over the control group. The best evidence that rehabilitation works comes from the 
stroke unit trials where most of the trials were trials of coordinated longer-term rehabilitation and not the hyperacute model of stroke units and the early supported discharge trials. ${ }^{17} 20$ There is evidence that very early mobilisation with high-intensity therapy after stroke may lead to poorer outcomes than physiotherapy protocols that use more frequent but less-intense spells of activity. ${ }^{56}$

\section{SUMMARY}

Progress in improving stroke care and outcomes has been rapid over the last 20 years but there remain many aspects where treatment is still ineffective. Investing in prevention should be at the forefront of efforts to reduce the burden and severity of stroke. Acute revascularisation through intravenous thrombolysis and thrombectomy needs to be made available to all who need it but not at the expense of the delivery of high-quality specialist acute and rehabilitation care for those who are not suitable or do not respond to revascularisation. Recovery can continue for many months and years after the acute event and the final message is not to give up too early and to remember that even in severely impaired people, there is nearly always some form of rehabilitation that can improve longer-term quality of life.

Contributors GM and AR contributed equally to the planning and writing of the review. AR submitted the paper and is responsible for the overall content as guarantor.

Funding The authors have not declared a specific grant for this research from any funding agency in the public, commercial or not-for-profit sectors.

Competing interests None declared.

Patient consent for publication Not required.

Provenance and peer review Commissioned; externally peer reviewed

\section{REFERENCES}

1 Sir William Osler the principles and practice of medicine: designed for the use of practitioners and students of medicine, volume 82. Page 1892.

2 Feigin VL, Forouzanfar MH, Krishnamurthi R, et al. Global and regional burden of stroke during 1990-2010: findings from the global burden of Disease Study 2010 The Lancet 2014;383:245-55.

3 Feigin VL, Nguyen G, Cercy K, et al. Global, regional, and Country-Specific lifetime risks of stroke, 1990 and 2016. N Eng/ J Med 2018;379:2429-37.

4 Stevens E, Emmett E, Wang Y, et al. The burden of stroke in Europe, 2017. Available: http://strokeeurope.eu/

5 Saka O, McGuire A, Wolfe CDA. Economic burden of stroke in England, 2005. Available: https://www.nao.org.uk/wp-content/uploads/2005/11/0506452_ economic_analysis.pdf

6 Adams HP, Bendixen BH, Kappelle LJ, et al. Classification of subtype of acute ischemic stroke. definitions for use in a multicenter clinical trial. TOAST. Trial of ORG 10172 in acute stroke treatment. Stroke 1993:24:35-41.

7 Kolominsky-Rabas P, Weber M, Gefeller O, et al. Epidemiology of ischemic stroke subtypes according to TOAST criteria: incidence, recurrence, and long-term survival in ischemic stroke subtypes: a population-based study. Stroke 2001;32:2735-40.

8 Romero JR, Morris J, Pikula A. Stroke prevention: modifying risk factors. Ther Adv Cardiovasc Dis 2008;2:287-303.

9 Joffres M, Falaschetti E, Gillespie C, et al. Hypertension prevalence, awareness, treatment and control in national surveys from England, the USA and Canada, and correlation with stroke and ischaemic heart disease mortality: a cross-sectional study. BMJ Open 2013;3:e003423.

10 Lewington S, Clarke R, Qizilbash N, et al. Age-specific relevance of usual blood pressure to vascular mortality: a meta-analysis of individual data for one million adults in 61 prospective studies. Lancet 2002;360:1903-13.

11 Wolf PA, Abbott RD, Kannel WB. Atrial fibrillation as an independent risk factor for stroke: the Framingham study. Stroke 1991;22:983-8.

12 Vermeer SE, Sandee W, Algra A, et al. Impaired glucose tolerance increases stroke risk in nondiabetic patients with transient ischemic attack or minor ischemic stroke. Stroke 2006:37:1413-7.

13 Sacco R, Adams R, Albers G, et al. Guidelines for prevention of stroke in patients with ischemic stroke or transient ischemic attack: a statement for healthcare professionals from the American Heart Association/American Stroke Association Council on stroke: co-sponsored by the Council on cardiovascular radiology and intervention: the American Academy of Neurology affirms the value of this guideline. Stroke 2006:37:577-617.

14 Fulcher J, O'Connell R, Voysey M, et al. Efficacy and safety of LDL-lowering therapy among men and women: meta-analysis of individual data from 174,000 participants in 27 randomised trials. Lancet 2015;385:1397-405.

15 Shinton R, Beevers $G$. Meta-analysis of relation between cigarette smoking and stroke. BMJ 1989;298:789-94.

16 National Institute for Health and Care Excellence. Technology Appraisal 210: Clopidogrel and modified-release dipyridamole for the prevention of occlusive vascular events [Online]. NICE, 2010c. Available: https://www.nice.org.uk/guidance/ TA210

17 Stroke Unit Trialists' Collaboration. Organised inpatient (stroke unit) care, 2013. Available: https://www.cochrane.org/CD000197/STROKE_organised-inpatient-strokeunit-care

18 Langhorne P, de Villiers L, Pandian JD. Applicability of stroke-unit care to low-income and middle-income countries. The Lancet Neurology 2012;11:341-8.

19 Langhorne P, Pollock A, Stroke Unit Trialists' Collaboration. What are the components of effective stroke unit care? Age Ageing 2002;31:365-71.

20 Cochrane. Services for reducing duration of hospital care in people with acute stroke, 2017. Available: https://www.cochrane.org/CD000443/STROKE_services-reducingduration-hospital-care-people-acute-stroke

21 National Institute of Neurological Disorders and Stroke rt-PA Stroke Study Group. Tissue plasminogen activator for acute ischemic stroke. N Engl J Med 1995:333:1581-8.

22 Cochrane review clot-dissolving drugs for treating ischaemic stroke in the early stages, 2014. Available: https://www.cochrane.org/CD000213/STROKE_clot-dissolving-drugstreating-ischaemic-stroke-early-stages

23 Flynn $\mathrm{D}$, Ford $\mathrm{GA}$, Rodgers $\mathrm{H}$, et al. A time series evaluation of the fast national stroke awareness campaign in England. PLOS ONE 2014;9.

24 Thomalla G1, Simonsen CZ1, Boutitie F, et al. For the wake-up Investigators. MRI-guided thrombolysis for stroke with unknown time of onset. N Eng/ J Med 2018:379:611-22.

25 Berkhemer OA, Fransen PS, Beumer D, et al. For the Mr CLEAN Investigators. A randomized trial of intraarterial treatment for acute ischemic stroke. N Engl J Med 2015:372:11-20.

26 Goyal M, Menon BK, van Zwam WH, et al. Endovascular thrombectomy after large-vessel ischaemic stroke: a meta-analysis of individual patient data from five randomised trials. The Lancet 2016;387:1723-31.

27 Nogueira RG, Jadhav AP, Haussen DC, et al. For the dawn Trial Investigators. thrombectomy 6 to 24 hours after stroke with a mismatch between deficit and infarct. N Eng/ J Med 2018;378:11-21.

28 Albers GW, Marks MP, Kemp S, et al. For the DEFUSE 3 Investigators. thrombectomy for stroke at 6 to 16 hours with selection by perfusion imaging. $N$ Eng/ J Med 2018:378:708-18.

29 Sentinel Stroke National Audit Porgramme. National Results - Clinical, 2017. Available: https://www.strokeaudit.org/results/Clinical-audit/National-Results.aspx

30 Bereczki D, Liu M, do Prado GF, et al. A systematic review of mannitol therapy for acute ischemic stroke and cerebral parenchymal hemorrhage. Stroke 2000;31:2719-22.

31 Vahedi K, Hofmeijer J, Juettler E, et al. For the DECIMAL, destiny, and HAMLET Investigators. Early decompressive surgery in malignant infarction of the middle cerebral artery: a pooled analysis of three randomised controlled trials. Lancet Neurol 2007:6:215-22.

32 Cruz-Flores S, Berge E, Whittle IR, et al. Surgical decompression for cerebral oedema in acute ischaemic stroke. Cochrane Database Syst Rev 2012;38.

33 Bray BD, Ayis S, Campbell J, et al. Associations between stroke mortality and weekend working by stroke specialist physicians and registered nurses: prospective multicentre cohort study. PLoS Med 2014;11:e1001705.

34 Bray BD, Ayis S, Campbell J, et al. Associations between the organisation of stroke services, process of care, and mortality in England: prospective cohort study. BMJ 2013;346:f2827.

35 Bray BD, Smith CJ, Cloud GC, et al. SSNAP collaboration. The association between delays in screening for and assessing dysphagia after acute stroke, and the risk of stroke-associated pneumonia. J Neurol Neurosurg Psychiatry 2016:pii: jnnp-2016313356.

36 Middleton S, McElduff $\mathrm{P}$, Ward J, et al. Implementation of evidence-based treatment protocols to manage fever, hyperglycaemia, and swallowing dysfunction in acute stroke (QASC): a cluster randomised controlled trial. The Lancet 2011:378:1699-706.

37 Kelly J, Rudd A, Lewis R, et al. Venous thromboembolism after acute stroke. Stroke $2001 \cdot 32 \cdot 262-7$

38 Kelly J, Rudd A, Lewis RR, et al. Venous thromboembolism after acute ischaemic stroke. A prospective study using magnetic resonance direct thrombus imaging Stroke 2004;35:2320-6.

39 Sandercock PAG, Counsell C, Kamal AK. Anticoagulants for acute ischaemic stroke. Cochrane Database Syst Rev 2008;4.

40 Dennis M, Sandercock PA, Reid J, CLOTS Trials Collaboration,. Et al. Effectiveness of thigh-length graduated compression stockings to reduce the risk of DVT after stroke (clots trial 1): a multicentre randomised controlled trial. Lancet 2009;373:1958-65. 
41 Dennis M, Sandercock PA, et al, The CLOTS (Clots in Legs or Stockings trial Collaboration. Thigh-length versus below-knee stockings for deep venous thrombosis prophylaxis after stroke. Ann Int Med 2010;153:553-62.

42 Dennis M, Sandercock P, Reid J, et al. Effectiveness of intermittent pneumatic compression in reduction of risk of deep vein thrombosis in patients who have had a stroke (clots 3): a multicentre randomised controlled trial. Lancet 2013;382:516-24.

43 Demchuk AM, Dowlatshahi D. Rodriguez-Luna D for the PREDICT/Sunnybrook ICH cta Study Group. Prediction of haematoma growth and outcome in patients with intracerebral haemorrhage using the CT-angiography spot sign (predict): a prospective observational study. Lancet Neurol 2012;11:307-14.

44 Anderson CS, Heeley E, Huang Y, et al. Rapid blood-pressure lowering in patients with acute intracerebral hemorrhage. N Engl J Med 2013:368:2355-65.

45 Qureshi Al, Palesch YY, Barsan WG, et al. ATACH-2 Trial investigators and the neurological emergency treatment trials Network. Intensive blood-pressure lowering in patients with acute cerebral hemorrhage. N Engl J Med 2016;375:1033-43.

46 Bower MM, Sweidan AJ, Shafie M, et al. Contemporary reversal of oral anticoagulation in intracerebral hemorrhage. Stroke 2019;50:529-36.

47 Parry-Jones A. Cutting delays in reversing anticoagulation after intracerebral haemorrhage: three key changes at a UK comprehensive stroke centre. BMJ Qual Improv Rep 2015;4. eCollection 2015.

48 Mendelow A, Gregson B, Fernandes H, et al. Early surgery versus initial conservative treatment in patients with spontaneous supratentorial intracerebral haematomas in the International surgical trial in intracerebral haemorrhage (STICH): a randomised trial. The Lancet 2005;365:387-97.

49 Mendelow AD, Gregson BA, Rowan EN, et al. Early surgery versus initial conservative treatment in patients with spontaneous supratentorial lobar intracerebral haematomas (STICH II): a randomised trial. The Lancet 2013;382:397-408.

50 Morris S, Hunter RM, Ramsay AIG, et al. Impact of centralising acute stroke services in English metropolitan areas on mortality and length of hospital stay: difference-indifferences analysis. BMJ 2014;349:g4757.
51 Ramsay AIG, Morris S, Hoffman A, et al. Effects of centralizing acute stroke services on stroke care provision in two large metropolitan areas in England. Stroke 2015;46:2244-51.

52 Hunter RM, Fulop NJ, Boaden RJ, et al. The potential role of cost-utility analysis in the decision to implement major system change in acute stroke services in metropolitan areas in England. Health Res Policy Sys 2018;16:23-6.

53 McKevitt C, Fudge N, Redfern J, et al. Self-reported long-term needs after stroke. Stroke 2011;42:1398-403.

54 Royal College of Physicians. National clinical guidelines for stroke (Intercollegiate stroke Working Party 5th edition 2016 Royal College Physicians, London, 2016. Available: https://www.strokeaudit.org/SupportFiles/Documents/Guidelines/2016National-Clinical-Guideline-for-Stroke-5t-(1).aspx

55 FOCUS Trial Collaboration. Effects of fluoxetine on functional outcomes after acute stroke (Focus): a pragmatic, double-blind, randomised, controlled trial. Lancet 2018.

56 Efficacy and safety of very early mobilisation within $24 \mathrm{~h}$ of stroke onset (AVERT): a randomised controlled trial. The Lancet 2015;386:46-55.

57 National Institute for Health and Care Excellence. Clinical Guideline 181 Cardiovascular disease: risk assessment and reduction, including lipid modification [Online], 2014c. Available: https://www.nice.org.uk/guidance/CG181

\section{Answers}

- Answers $a, b$ and $d$ true; $c$ and $e$ false

- Answers $a, b, c$ and e true; $d$ false

- Answers e true; $a, b, c$ and d false

- Answers a and e true; $b, c$ and $d$ false

- Answers $d$ and e true; $a, b$ and c false 